ARTÍCULO DE REVISIÓN

\title{
REVALORIZACIÓN DE PROPIEDADES PLANTA Y EQUIPO (PPYE) UNA APLICACIÓN DESDE ECUADOR
}

\section{REVALUATION OF PROPERTY, PLANT AND EQUIPMENT (PP\&E) AN APPLICATION FROM ECUADOR}

\author{
Gonzalo Junior Chávez Cruz \\ Doctor en Ciencias Contables (UNMSM) - Docente en la Universidad Técnica de Machala - Ecuador \\ Email: gonzach123@hotmail.com (Autor corresponsal) \\ John Alexander Campuzano Vásquez \\ Doctor en Economía y Estrategia Empresarial (UDC - España)- Docente en la Universidad Técnica de Machala - Ecuador \\ Wilton Eduardo Romero Black \\ Doctorado en Ciencia y Tecnología Ambiental (UDC - España)- Docente en la Universidad Técnica de Machala - Ecuador
}

[Recibido: 16/10/2016 Aceptado: 12/01/2017]

\section{RESUMEN}

Este artículo trata sobre la importancia de la aplicación de las NIIF (Normas Internacionales de Información Financiera) en el Ecuador utilizando el grupo de cuentas denominado: revalorización de propiedades, planta y equipo (PPyE). Para ello, se analiza conceptualmente como se deben reportar y registrar los estados financieros a valor razonable, considerando algunos países del mundo en donde hay avances significativos en este tema, se revisan modelos y técnicas de revaluación que permiten entender los procedimientos técnicos. Metodológicamente se utilizó información de una empresa local de transporte, que permitió crear un plan de cuentas para el registro y afectación contable de una revaluación de edificios, además, se ejemplifica mediante el uso de un gráfico de procesos y tablas explicativas como realizar el ajuste utilizando la normativa de las NIIF. Los resultados muestran que no se está registrando adecuadamente el valor de edificios en los estados financieros de la empresa al no utilizar la NIIF 16, que es la guía para el uso correcto de una revaluación de una cuenta que pertenece a propiedad, planta y equipos. Finalmente, se considera la importancia de asesorar adecuadamente a los administradores de empresas sobre este tipo de revaluación, por las implicaciones financieras y tributarias que existen.

\section{PALABRAS CLAVE}

Revaluación, NIC 16, políticas contables, propiedad planta y equipo, estados financieros.

\begin{abstract}
This article discusses the importance of the application of IFRS (International Financial Reporting Standards) in Ecuador using the group of accounts called: Property, Plant and Equipment (PP\&E) revaluation. To do this, we analyze conceptually how financial statements should be reported and recorded at fair value, considering some countries in the world where there is significant progress in this area, we review models and revaluation techniques that allow us to understand technical procedures. Methodologically, we used information from a local transport company, which allowed us to create a chart of accounts to recording and accounting the revaluation of buildings, in addition, this is exemplified using a process chart and explanatory tables of how to make the adjustment using the regulations of the IFRS. The results show that the value of buildings in the company's financial statements is not being properly recorded by not using IFRS 16, which is the guide for the correct use of a revaluation of an account belonging to Property, Plant and Equipment. Finally, the importance of properly advising the managers of companies on this type of revaluation is considered, due to the existing financial and tax implications.
\end{abstract}

\section{KEYWORDS}

Revaluation, IAS 16, accounting policies, plant and equipment property, financial statements.

Como Citar: Chávez, G., Campuzano, J., y Romero, W. (2017). Revalorización de Propiedades de Planta y Equipo (PPyE) una aplicación desde Ecuador. Quipukamayoc, 25(47), 85 - 93. doi: http://dx.doi.org/10.15381/quipu.v25i47.13806

\footnotetext{
(c) Los autores. Este artículo es publicado por la Revista Quipukamayoc, Universidad Nacional Mayor de San Marcos. Este es un artículo de acceso abierto, distribuido bajo los términos de la Licencia Creative Commons Atribución-NoComercial-Compartirlgual 4.0 Internacional.(http://creativecommons.org/licenses/by-nc-sa/4.0/), que permite el uso no comercial, distribución y reproducción en cualquier medio, siempre que la obra original sea debidamente citadas.
} 


\section{INTRODUCCIÓN}

La presencia de las normas internacionales de información financiera es cada vez más fuerte en el Ecuador, sus orientaciones están ayudando a revelar la situación real de empresas y personas.

Es tanta la urgencia de comenzar a trabajar en su aplicación, que muchas veces se ignora cómo deben reconocerse cuentas como propiedades, plantas y equipos (PPyE) y el análisis que hay que hacer para su correcto registro y posteriores beneficios económicos si fuera el caso. En nuestro país a partir de la adopción de las NIIF en el año 2010, todas las entidades controladas por la Superintendencia de Compañías (SC) deben reportar obligatoriamente en sus estados financieros la cuenta propiedad, planta y equipo aplicando la NIC 16.

Por lo que ya hay un mandato legal que debe ser aplicado a los activos no corrientes. Debe mencionarse, que a pesar de ser obligatorio el registro, aún no logra establecerse por completo en el país por su costo y por las implicaciones tributarias que tienen para diferentes agentes económicos; de ahí, que la importancia de conocer el procedimiento contable para su aplicación.

Para proceder a su contabilización o registro, hay que tener claridad en los conceptos utilizados; por ello, se mencionan los más importantes para saber cómo proceder a revalorizar activos en la actividad empresarial.

Hay trabajos teóricos como el de Castellanos (2015), que señala el aporte de las NIIF en los países que las han adoptado, ha servido para que las empresas reporten y preparen información estandarizada utilizando bases teóricas de valor razonable para la valoración de activos y pasivos.
En el caso de empresas españolas, se da cuenta que el modelo de registro de las inversiones inmobiliarias mediante valor razonable (VR como se conocerá), reporta resultados más relevantes en los estados financieros (Vicente, Ramírez, y Molina, 2013). Con esta afirmación discrepa Linsmeier (2013), tomando partido por el costo histórico como reporte de mayor fiabilidad, antes que el VR, con lo que se puede avizorar una doble consideración al momento de estimar valores de activos y pasivos.

Tomando el aporte de Sosa (2014), para quién el modelo de costo histórico tiene fuertes críticas en los últimos diez años, por no presentar la realidad de las empresas debido a la alta inflación que experimentaban países, a más de no responder a los cambios rápidos que vive el mundo, por lo que lo considera un modelo estático frente a las cambiantes necesidades de los usuarios.

Chiquiar (2010), indica que no solo es de tomar partido por un determinado procedimiento, además en el cálculo del VR se dice que este debe ser manejado con habilidad e ingenio por parte del profesional que realiza el avalúo, el que debe valerse de las matemáticas para usar técnicas de medición que sean sometidas a criterios y experiencia como condición fundamental $y$ necesaria.

Algunas experiencias locales en Ecuador, demuestran problemas de peritaje, por la falta de consenso entre peritos que manejan sus propias técnicas, a su vez las empresas creen conocer mejor el mercado y cada dueño piensa en las mejores razones para justificar su valor. Lo relevante, es en todo caso, conocer el bien a tasar, mantener la objetividad y finalmente conocer el medio y el momento.

Un trabajo de Latriidis y Kilirgiotis
(2012), refiriéndose a México, señala que las normas internacionales de contabilidad para el sector público (IPSAS) establecen los requerimientos mínimos para el registro a VR de PPyE y que estas se apoyan en el valor del costo histórico del activo menos el cálculo de su depreciación Y como hecho relevante indican que el VR de PPyE, ayuda a los inversionistas, entidades financieras y analista financieros a conocer la salud financiera sobre el desempeño futuro de una empresa.

García y Yaguana (2015), documentan en Ecuador en base a la resolución $\mathrm{N}^{\circ}$ SC.ICI.CPAIIFRS.G.11.015 de las NIIF para PYMES, en la aplicación de la sección 35 , la existencia de una nueva normativa para la medición del valor razonable o reevalúo, para el caso exclusivo de bienes inmuebles, la que toma el VR del impuesto predial, o en su defecto, el mismo puede ser tomado mediante un nuevo avaluó realizado por un perito calificado por la Superintendencia de Compañías, el que debería demostrar la forma como calculó ese valor. En todo caso luego de revisar antecedentes, se observa opiniones divididas en la utilización del costo histórico y el VR, y más de un procedimiento que será evaluado más adelante.

\section{Modelos de Revaluación}

Si hablamos de modelos de revaluación hay que considerar a Yamamoto (2014) y su investigación en Reino Unido, que explica la utilización del modelo por parte de empresas de bienes raíces que quieren mostrar un valor real de los edificios y terrenos en los mercados activos, diferencia con el sector de las industrias, transportes, energía, servicios, comunicación, comercio los que utilizan el modelo del costo histórico. Vicente, Ramírez, y Molina (2013), encuentran en España iguales aplicaciones a las del Reino Unido, por lo que las inversiones 
inmobiliarias mayoritariamente han sido registradas bajo el modelo de VR.

En esta línea Latriidis y Kilirgiotis (2012), aportan otro sector al uso del $\mathrm{VR}$, el que se ubica en la exportación e importación por la necesidad de captar recursos que le permitan financiar sus negocios en el extranjero.

Por lo que se puede plantear la siguiente interrogante: ¿cuál es el método sugerido o conveniente para que una empresa pueda revalorizar sus activos y que su impacto sea el mínimo en los estados financieros?

Betancur (2013), hace un aporte, al referirse a los métodos para revisar activos, trae a revisión la necesidad de aplicar homogeneización, al que lo califica de subjetivo, por la discrecionalidad que recae en el perito de poner valores a las distintas variables. Sin embargo, hay como disminuir la subjetividad de la homogeneización al tomar en cuenta las ciencias como la estadística, economía y las matemáticas, como señalan otros autores.

El método de homogeneización mencionado por Betancur usa la Norma Argentina TTN 3,0, para quitar la subjetividad a la homogeneización, utilizando coeficientes que se encuentre entre 0,70 a 1,30 y en todo caso precisa, que si el valuador llegara a utilizar un valor que no se encuentre en los rangos mencionados, se pide que se indique en detalle en el respectivo documento de revaluación.

Hay indicadores que usan, características del entorno, existencia de garaje, ascensor, entorno urbano, instalaciones y amueblamiento, antigüedad del edificio, calidad de edificio, calidad de edificio entre otros. Y son, una ayuda importante para determinar el valor final del activo o del pasivo.

Tomando el aporte de la firma de auditoría Deloitte (2015) que se refiere a la NIC 16, este señala que las empresas son las que escogen el modelo de valoración en función de sus políticas contables. Aquí vale hacer una precisión en el tamaño de las empresas, las medianas y grandes habitualmente optarán por revalorizar sus activos $\mathrm{y}$ pasivos, mientras que las pequeñas tal vez por sus necesidades no tengan una obligación urgente.

\section{Técnicas de Revaluación}

Para la fundación IFRS (2015), en la sección 11, párrafo 11,28 sostiene que en las técnicas de valoración deben incluirse las transacciones de mercado recientes para activos iguales o idénticos entre partes interesadas $y$ que estén debidamente informadas, además que actúen en condiciones de independencia mutua.

Por otro lado, si hubiera una técnica utilizada por las partes interesadas para fijar el precio de los activos y se lograra demostrar que este instrumento entrega estimaciones fiables de los precios en transacciones reales de mercado, la entidad podrá utilizar esta técnica. Por lo que no estaríamos trabajando con técnicas fijas, sino variables de acuerdo a las realidades del entorno.

Otra técnica utilizada para revalorar un activo es la del Método de Comparación Directa o Método de Mercado, cuya teoría se basa en la comparación del activo con otro lo más similar posible que relacione las características intrínsecas y extrínsecas, también señala que otra técnica utilizada es la de Homogeneización de datos que anteriormente ha sido mencionada, bajo esta técnica es necesario encontrar las similitudes referenciales con respecto al inmueble a revaluar y el referencial a tomar en cuenta es: fecha de registro, número de tomo en que quedo registrado el inmueble, tipología del inmueble, localización, ubicación, precio tasado o valor en el registro, área y que para su aplicación de esta técnica existen dos procedimientos para hacerlo:

Homogenización tradicional o semiempírica.- Se la aplica con la utilización de fórmulas, pero tiene sus limitaciones al no poder ser aplicada para todos los estudios al tener que utilizar los siguientes criterios:

Mediante el criterio de actualización, contemporaneidad o ajuste por tiempo, pueden obtenerse o no datos en una fecha muy cercana al avalúo, en todo caso deben ser actualizadas y con la ayuda de las matemáticas financiera, se los trae a valor presente, donde el valor actual es:

$$
\frac{\operatorname{Va}=\operatorname{Vr}(1+\mathrm{i}) \mathrm{n}}{\mathrm{X}}
$$

Donde:

$\mathrm{Va}=$ Valor actualizado a la fecha de avaluó del precio unitario del referencial

$\mathrm{Vr}=$ Valor del precio unitario del referencial para la fecha del registro

$\mathrm{i}$ = Tasa de incremento de los precios de los inmuebles en el periodo $\mathrm{n}=$ Numero de periodos considerados

Y donde el Factor de corrección por Fecha es igual:

$\mathrm{FcF}=(1+\mathrm{i}) \mathrm{n}$

Aplicando el criterio de ajuste por área, se conoce que el precio unitario de un bien inmueble es inversamente proporcional a la magnitud de su área, en su gran mayoría cuando mayor es el área tenemos un menor precio unitario y viceversa, es muy importante conseguir referenciales cuyas áreas no logren superar $+/-30 \%$, para realizar una comparación con el área del inmueble que se va a revaluar y en todo caso si no se logra obtener los referenciales podrán aplicarse las siguientes fórmulas: 
Fórmula de Meyer: citada por Betancur (2013), plantea dos fórmulas y su aplicación depende si el área referencial es mayor o menor que la del in-

mueble motivo de estudio y en todo caso estas fórmulas no debieran ser aplicadas para superficie muy grandes:

\section{Tabla 1.}

\section{Formula de Meyer}

Fuente: Tomado de Betancur (2013) /Elaboración de autores

\begin{tabular}{cc}
$\begin{array}{c}\text { Área del referencial es menor que el } \\
\text { área del inmueble a revaluar }\end{array}$ & $\begin{array}{c}\text { Área del referencial es mayor que el } \\
\text { área del inmueble a revaluar }\end{array}$ \\
$\mathrm{FcA}=(1-($ Ainm/Aref $) * \mathrm{X})+\mathrm{X}$ & $\mathrm{FcA}=(1+($ Ainm/Aref $) * \mathrm{X})-\mathrm{X}$ \\
\hline
\end{tabular}

Donde:

FcA = Factor de corrección por Área

Aref $=$ Área del referencial

Aim = Área del inmueble objeto del

reevaluó

$\mathrm{X}=$ Porcentaje de afectación entre el $5 \%$ y el $10 \%$

Tabla 2.

\section{Formula de Empírica}

Fuente: Tomado de Betancur (2013) /Elaboración de autores

Si la diferencia entre el área referencial Si la diferencia entre el área refereny el área del inmueble es menor o igual a $30 \%$ cial y el área del inmueble es mayor a $30 \%$

$\mathrm{FcA}=($ Aref $/$ Ainm $) \wedge 0.25$ $\mathrm{FcA}=($ Aref $/$ Ainm $) \wedge 0.30$

Tabla 3 .

\section{Plan de Cuentas}

Fuente: Elaboración de autores

\begin{tabular}{ll} 
1. & ACTIVOS \\
1.1. & ACTIVO CORRIENTE \\
1.2. & ACTIVOS NO CORRIENTES \\
1.2.1. & PROPIEDADES, PLANTA Y EQUIPO \\
\hline 1.2.1.01. & Terrenos \\
\hline 1.2.1.02. & Ajuste Acumulado por Revaluación de Terrenos \\
\hline 1.2.1.03. & Edificios \\
\hline 1.2.1.04. & Ajuste Acumulado por Revaluación de Edificios \\
\hline 1.2.2. & AMORTIZACIÓN ACUMULADA DE PPyE \\
\hline 1.2.2.01. & Amortización Acumulada de Edificios \\
\hline 1.2.2.02. & Ajuste Acumulado por Revaluación de Edificios \\
\hline 1.2.7. & ACTIVOS POR IMPUESTOS DIFERIDOS \\
\hline 1.2.7.01. & Por diferencias Temporarias Deducibles de Terrenos \\
\hline 1.2.7.02. & Por diferencias Temporarias Deducibles de Edificios \\
\hline 2. & PASIVO \\
\hline 2.1. & PASIVO CORRIENTE \\
2.2. & PASIVO NO CORRIENTE \\
2.2.9. & PASIVOS POR IMPUESTOS DIFERIDOS \\
\hline
\end{tabular}

\section{Políticas contables}

Las políticas contables según Monterrey, J. (1998) citado por Suardi, et. al (2015), la elección de una política contable es eficiente si le permite a la entidad optimizar las decisiones basados en los reportes contables y la considera oportunista, cuando sus administradores seleccionan unas políticas que favorezcan a sus intereses y concluye indicando, que si se toma como política el (VR) permitirá brindar información más relevante y confiable a los lectores de sus estados financieros. También hay que señalar, que las entidades pueden realizar cambios en las políticas contables debido a dos circunstancias, la primera, por un cambio en esta norma y la otra porque los estados financieros suministren información relevante sobre los efectos de las transacciones, según consta en el párrafo 10 de la sección 10.8 de las NIIF.

\section{Enfoque de Caso}

Se analizan las implicaciones que tiene una revaluación técnica en lo tributario y financiero, considerando la aplicación correcta de la NIC 16 PPyE. Para ello se procedió a utilizar información real de la empresa Móvil S.A de transporte, que permitió crear un plan de cuentas para el registro de las afectaciones contables en la revaluación de un edifico, en base al análisis del factor de corrección; el criterio de ajustes por áreas hasta lograr obtener un referencial de +/- 30\% que está presente en la fórmula de Meyer, procediendo al registro en el diario general como lo exige la norma internacional. Como un aporte a la identificación correcta de las cuentas que son parte de PPyE se presentan las cuentas que deben ser afectadas considerando un activo revaluado para el caso de estudio.

Chávez, Campuzano, Alvarado (2015) 
afirman que las cuentas que conforman los activos de una empresa se debitaran por el costo de su adquisición, al que se debe incluir las mejoras y los ajustes por variaciones catastrales o comerciales, registrándolos de manera individual y siendo controlados por separado todo incremento y serán acreditados por la venta total o parcial de la PPyE, el gasto por depreciación mensual $o$ anual del activo.

En el Primer Caso, presentando el valor neto del activo, cuando la depreciación es eliminada contra el valor en libros. La empresa Móvil S.A. posee un edificio cuyo costo histórico es $\$$ 145 00,00, y su valor en libros al 31 de Diciembre del 2015 es \$ 116 000,20, su depreciación acumulada es de $\$$ 28 999,80. La administración de esta empresa le ha dado un correcto mantenimiento por lo que no ha sufrido deterioro el edificio.

Tabla 4.

\begin{tabular}{ll}
\hline 2.2.9.01. & Diferencias Temporales de Terrenos \\
\hline 2.2.9.02. & Diferencias Temporales de Edificios \\
\hline 3.1. & PATRIMONIO \\
3.1.4. & SUPERÁVIT POR REVALUACIÓN DE PPYE \\
\hline 3.1.4.01. & Superávit por Revaluación Terrenos \\
\hline 3.1.4.02. & Superávit por Revaluación Edificios \\
\hline 5.1. & COSTOS Y GASTOS \\
5.1.1. & COSTOS \\
5.1.2. & GASTOS \\
5.1.2.6. & GASTOS DEPRECIACIÓN NO ACELERADA DE PPyE \\
\hline 5.1.2.6.01. & Gasto Depreciación de Edificio \\
5.1.2.7. & GASTO AJUSTE ACUMULADO REVALUACIONES DE \\
\hline 5.1.2.7.01. & PPyE \\
\hline
\end{tabular}

En el proceso de adopción de NIIF este activo no fue objeto de revaluación por lo que se contrata un perito calificado por la Superintendencia de Compañías, para que realice el estudio de este activo.
Luego del peritaje el Edificio ha sido revaluado en un valor de mercado por $\$ 147740,30$. Su vida útil es de 20 años y fue terminado de construir el 28/12/2011 por lo que la empresa lo ha utilizado 4 años.

Datos para calcular la depreciación periodo 2015

Fuente: Elaboración de autores

\begin{tabular}{|c|c|c|c|}
\hline Costo Histórico & 145000,00 & Valor residual & 1 \\
\hline Vida útil & 20 años & Depreciación acumulada & 28999,80 \\
\hline \multicolumn{2}{|c|}{ Depreciación anual $=\frac{\text { Costo histórico }- \text { valor residual }}{\text { Vida útil }}$} & \multicolumn{2}{|c|}{$\begin{array}{l}\text { Como han transcurrido los periodos: 2012, 2013, } 2014 \text { y } 2015 \\
\text { la depreciación acumulada será: }\end{array}$} \\
\hline \multicolumn{2}{|c|}{ Depreciación anual $=\underline{145000,00-1}=7$ 249,95 } & Depreciación $=7249,95 x$ & \\
\hline
\end{tabular}

Tabla 5.

Calculo de valor en libros según cálculos de la depreciación y de revaluación

Fuente: Elaboración de autores

\begin{tabular}{lllc}
\multicolumn{1}{c}{ Cálculo de valor en libros } & \multicolumn{2}{c}{ Cálculo de revaluación } \\
Edificio & 145000,00 & Valor mercado actual & 147740,30 \\
\hline Amortización acumulada & 28999,80 & Valor en libros & 116000,20 \\
\hline Valor en libros & 116000,20 & Revaluación & 31740,10 \\
\hline
\end{tabular}

Tabla 6.

Valor Razonable

Fuente: Elaboración de autores

\begin{tabular}{lc}
\multicolumn{1}{c}{ Valor razonable } & Valor \\
Nuevo costo histórico & 176740,10 \\
\hline Amortización acumulada & 28999,80 \\
\hline Valor Razonable en libros & 147740,30 \\
\hline
\end{tabular}

Cálculo de la depreciación ejercicio Vida útil 20 años 2016

Valor residual

1

Nuevo valor de edificio 176 740,10 Amortización acumulada 28 999,80

Depreciación anual $=\underline{\text { Valor razonable }- \text { valor residual }}$

Vida útil restante

Depreciación anual $=\frac{147740,30-1}{16}=9233,71$ 
Tabla 7.

Cálculo de la depreciación

Fuente: Elaboración de autores

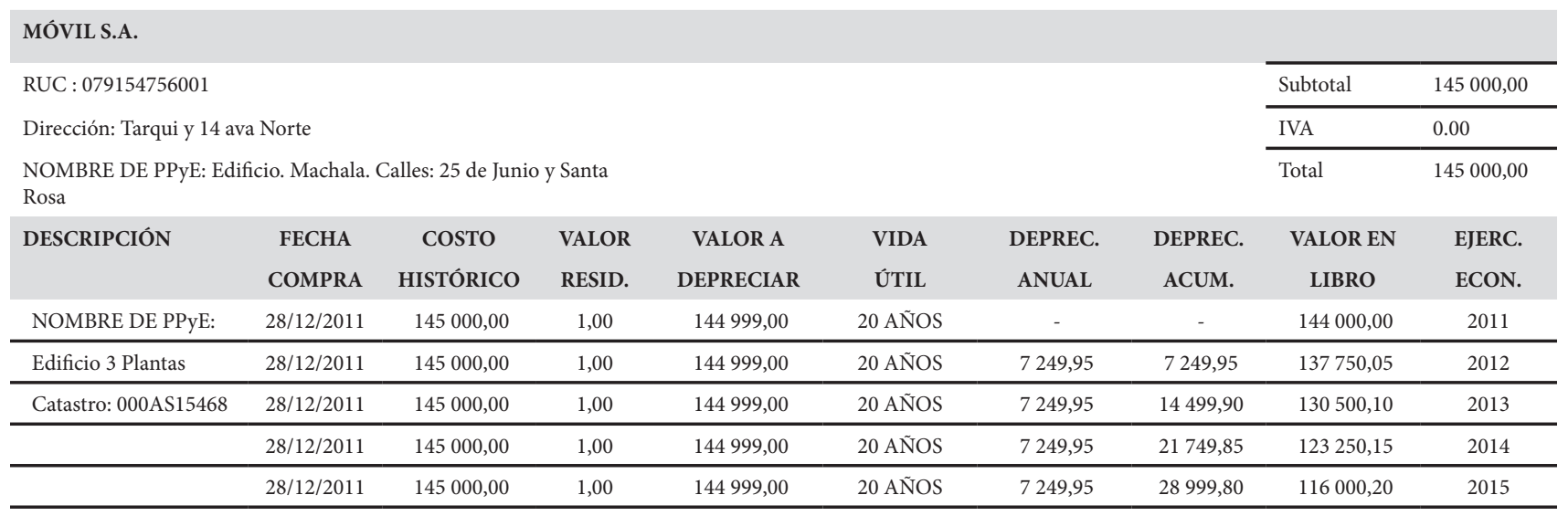

Tabla 8 .

Contabilización del valor neto del activo

Fuente: Elaboración de autores

\begin{tabular}{|c|c|c|c|c|c|}
\hline \multirow[b]{2}{*}{ Fecha } & \multicolumn{3}{|c|}{ Libro Diario General } & \multirow[b]{2}{*}{ Debe } & \multirow[b]{2}{*}{ Haber } \\
\hline & CÓDIGO & Detalle & Parcial & & \\
\hline \multicolumn{6}{|c|}{1} \\
\hline \multirow[t]{6}{*}{$31 / 12 / 2015$} & 1.2 .2 & AMORTIZACIÓN ACUMULADA DE PPyE & & 28999,80 & \\
\hline & 1.2.2.01 & Amortización Acumulada de Edificio & 28999,80 & & \\
\hline & 1.2.1 & PROPIEDADES, PLANTA Y EQUIPO & & & 28999,80 \\
\hline & 1.2.1.03 & Edificios & 28999,80 & & \\
\hline & & P/R Eliminación Amortización de PPyE & & & \\
\hline & & 2 & & & \\
\hline \multirow[t]{6}{*}{$31 / 12 / 2015$} & 1.2.1 & PROPIEDADES, PLANTA Y EQUIPO & & 31740,10 & \\
\hline & 1.2.1.04 & Ajuste Acumulado por Revaluación Edificios & 31740,10 & & \\
\hline & 3.1.4 & SUPERÁVIT POR REVALUACIÓN DE PPYE & & & 31740,10 \\
\hline & 3.1.4.02 & Superávit por Revaluación de Edificios & 31740,10 & & \\
\hline & & P/R. Revaluación de edificio según peritaje & & & \\
\hline & & 3 & & & \\
\hline \multirow[t]{5}{*}{$31 / 12 / 2015$} & 3.1 .4 & SUPERÁVIT POR REVALUACIÓN DE PPYE & & 6982,82 & \\
\hline & 3.1.4.02 & Superávit por Revaluación de Edificios & 6982,82 & & \\
\hline & 2.2 .9 & PASIVOS POR IMPUESTOS DIFERIDOS & & & 6982,82 \\
\hline & 2.2.9.02 & Diferencias Temporales de Edificios & 6982,82 & & \\
\hline & & P/R. Cálculo de Impuesto Renta Diferido 22\% & & & \\
\hline
\end{tabular}




\begin{tabular}{|c|c|c|c|c|c|}
\hline & & 4 & & & \\
\hline \multirow[t]{6}{*}{$31 / 12 / 2016$} & 5.1.2.6 & GASTO DEP. NO ACELERADA DE PPyE & & 7249,95 & \\
\hline & 5.1.2.6.01 & Gasto Depreciación de edificio & 7249,95 & & \\
\hline & 1.2 .2 & AMORTIZACIÓN ACUMULADA DE PPyE & & & 7249,95 \\
\hline & 1.2.2.01 & Amortización Acumulada de Edificio & 7249,95 & & \\
\hline & & P/R. Gasto por depreciación ejercicio 2016 & & & \\
\hline & & Total & & 74972,67 & 74972,67 \\
\hline
\end{tabular}

Para el asiento 1 de la empresa Móvil Sociedad Anónima las variaciones son registradas en el diario general cuando se elimina la amortización acumulada de edificio contra la PPyE (Edificio). En el 2 se incrementa el valor del edificio haciendo un debito en la cuenta de ajuste acumulado por re- valuación edificios y su impacto se ve reflejado en una cuenta de patrimonio llamada superávit por revaluación de propiedad planta y equipo. En el registro 3 se registra un pasivo en el crédito por diferencias temporales de edificios contra un debito en superávit por revaluación de edificios, en la transacción 4 , se reconoce el desgaste sufrido y su valor es debitado y se acredita la amortización acumulada de edificio.

Para el caso 2, presentando el factor de proporcionalidad

Tabla 9.

Superávit por revaluación

Fuente: Elaboración de autores

\begin{tabular}{cccc} 
Costo histórico del edificio & 145000,00 & Depreciación acumulada & 28999,80 \\
Valor Residual & 1,00 & Perito valor razonable & 147740,30 \\
\hline Vida Útil & 20 años & Valor en libros & 116000,20 \\
\hline Valor en libros & 116000,20 & Revaluación & 31740,10 \\
\hline
\end{tabular}

\section{Cálculo del factor proporcional}

Factor proporcional $=$ Revaluación $=\underline{147740,30}=1,27362108$

Valor en libros 116000,20

Costo histórico

$$
\begin{array}{r}
145000,00 \times 1,27362108=184675,06 \\
28999,80 \times 1,27362108=\frac{36934,76}{147740,30}
\end{array}
$$

Depreciación acumulada $28999,80 \times 1,27362108=\underline{36934,76}$

Valor según libros

El valor razonable es \$ 147740,30

Tabla 10 .

Superávit por revaluación

Fuente: Elaboración de autores

\begin{tabular}{crrr} 
& \multicolumn{1}{c}{ Inicial } & Nueva valor & Variación \\
Costo histórico & 145000,00 & 184675,06 & 39675,06 \\
\hline Depreciación acumulada & 28999,80 & 36934,76 & 7934,96 \\
\hline Valor según libros & 116000,20 & 147740,30 & 31740,10 \\
\hline
\end{tabular}


Tabla 11.

Contabilización por el método del factor de proporcionalidad

Fuente: Elaboración de autores

\begin{tabular}{|c|c|c|c|c|c|}
\hline & & $\begin{array}{c}\text { Libro Diario General } \\
\text { Del } 1 \text { de Enero del } 2015 \text { al } 31 \text { de Diciembre }\end{array}$ & 2015 & & \\
\hline Fecha & CÓDIGO & Detalle & Parcial & Debe & Haber \\
\hline & & 1 & & & \\
\hline $31 / 12 / 2015$ & 1.2.1 & PROPIEDADES, PLANTA Y EQUIPO & & 39675,06 & \\
\hline & 1.2.1.04 & Ajuste Acumulado por Revaluación Edificios & 39675,06 & & \\
\hline & 5.1.2.6 & GASTO DEP. NO ACELERADA DE PPyE & & & 7934,96 \\
\hline & 5.1.2.6.01 & Gasto Depreciación de edificio & 7934,96 & & \\
\hline & 3.1 .4 & SUPERÁVIT POR REVALUACIÓN DE PPYE & & & 31740,10 \\
\hline & 3.1 .4 .02 & Superávit por Revaluación de Edificios & 31740,10 & & \\
\hline & & P/R. Revaluación PPyE factor proporcionalidad & & & \\
\hline & & 2 & & & \\
\hline $31 / 12 / 2015$ & 3.1 .4 & SUPERÁVIT POR REVALUACIÓN DE PPYE & & 6982,82 & \\
\hline & 3.1 .4 .02 & Superávit por Revaluación de Edificios & 6982,82 & & \\
\hline & 2.2 .9 & PASIVOS POR IMPUESTOS DIFERIDOS & & & 6982,82 \\
\hline & 2.2 .9 .02 & Diferencias Temporales de Edificios & 6982,82 & & \\
\hline & & P/R. Cálculo de Impuesto Renta Diferido 22\% & & & \\
\hline & & Total & & 46657,88 & 46657,88 \\
\hline
\end{tabular}

Las variaciones del valor de la PPyE se obtiene dividiendo el nuevo valor revaluado para el valor en libros; este resultado es multiplicado por el costo histórico y también por la depreciación acumulada y la variación obtenida será el resultado del nuevo valor menos el valor inicial; los valor obtenidos son registrados en el diario general y al final se plantea un diagrama de flujo para la revaluación de los activos de la empresa.

\section{CONCLUSIONES}

Al aplicar la NIC 16, PPyE, párrafo 28 y 42 , NIC 8 , políticas contables, la gerencia de la empresa Móvil S.A., escogió el modelo de registro revaluación de PPyE y las políticas de valoración, depreciación, vida útil y luego de haber revalorizado el activo no financie-

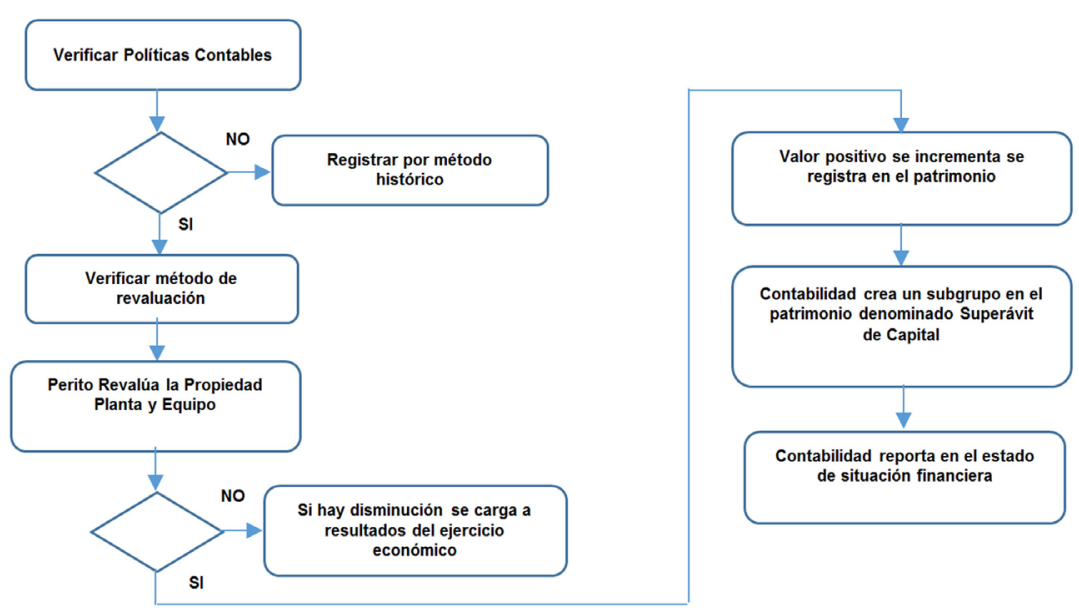

Figura 1. Diagrama de proceso para la revaluación de la Propiedad Planta y Equipos Fuente: Elaboración de autores

ro (edificio) en su valor de mercado por un perito independiente cualificado por la Superintendencia de Compañías en el ejercicio económico 2015, se determinó que hubo un incremento de \$ 37740,10 , siendo positivo, el departamento contable registró este incremento en el grupo del patrimo- 
nio acreditando a la cuenta ajuste acumulado por revaluación de edificio. Este aumento produjo un incremento en el patrimonio afectando la cuenta superávit por revaluación de edificio, en nuestro país dicho incremento debe registrarse como un pasivo por impuesto diferido por $\$ 6982,82$ y en su cuarto asiento se reconoce el gasto por depreciación acumulada que es de \$ 7249,95 .

Para el segundo caso se aplica la fórmula del factor de proporcionalidad dichas variaciones se registran en el diario general como se muestra en la Tabla 9.

Al final podemos concluir que es importante que los colegas contadores propongan y asesoren a los administradores de las empresas ecuatorianas sobre la importancia de reportar a valor razonable los activos no financieros.

\section{REFERENCIAS BIBLIOGRÁFICAS}

Betancur, D. D. (2013). Procedimiento de homogeneización por factores mediante pendientes. Trabajo presentado en el I Encuentro de Panamericano de Evaluación de Intangibles. Encuentro llevado a cabo en el XXVIII Congreso Panamericano de Valuación UPAV, Cartagena, Colombia.

Castellanos, H. (2015). Medición de activos no financieros. Un análisis de los elementos que intervienen en la decisión de la gerencia al optar por el modelo del valor razonable. Cuadernos de Contabilidad, 16 (40), pp.4171.

Chávez, G., Campuzano, J., \& Alvarado, F. (2015) Contabilidad Intermedia. Machala: Ediciones UTMACH.

Chiquiar, W. (Noviembre de 2010). El Modelo de Revaluación en las Normas Internacionales de Información Financiera NIC-NIIF. Casos Aguas Andinas \& NH Hoteles. En A. Barbieri (Presidencia). IV Congreso Internacional de Economía y Gestión ECON 2010, Buenos Aires, Argentina.

Deloitte. (2015). Guía Rápida IFRS. Recuperado de https://www2.deloitte. com/content/dam/Deloitte/es/Documents/servicios- financieros/Deloitte_ES_Servicios_Financieros_Guiarapida-IFRS.pdf

García, H. \& Yaguana, A. (2015). Análisis integral asociado a la aplicación de las NIIFS en las PYMMES; caso: Salcon Cía Ltda. (Tesis para optar el título de Contador Público Auditor). Universidad de Cuenca, Cuenca, Ecuador.

Fundación de Estándares Internacionales de Reportes Financieros. (2015). Modificaciones de 2015 a la Norma Internacional de Información Financiera para Pequeñas y Medianas Entidades. Norma NIIF para la PYMES. Londres: IFRS Foundation Publications Department.
Latriidis, G., y Kilirgiotis, G. (2012). Incentives for fixed asset revaluations: The UK evidence. Journal of Applied Accounting Research, 13(1), pp. 5-20.

Linsmeier, T. (2013). A Standard setters framework for selecting between fair value and historical cost measurement attributes: a basis for discussion of "Does fair valúe accounting for non-financial assets pass the market test?". Accinting Studies, 18(1), pp. 776-782.

Sosa, E. (2014). El auge del modelo del valor razonable en las normas internacionales de información financiera. Pensamiento Actual. 14(22), pp. 85-98

Suardi , D., Bertolino, G., Díaz , T., Chiurchiu, A., Galante , E., \& Pozzi, N. (2015). La elección de la política contable en el marco de la NIC 16: "Propiedad, planta y equipo". Trabajo presentado en la Vigésima Jornada "Investigaciones en la Facultad" de Ciencias Económicas y Estadisticas, Rosario, Argentina.

Vicente, M., Ramírez, J., \& Molina, H. (2013). Inversiones inmobiliarias: la elección contable valor razonable versus coste en los grupos cotizados españoles. Cuaderno de Contabilidad, 14(34), pp. 25-51.

Yamamoto, T. (2014). Fair Value of Investment Property and independent Appraisers: The Experience in the UK and Japan. Academic Journal, 82(2), pp. 138-154. 